\title{
La lodyans caricaturale dans la comédie humaine haïtienne de Gary Victor ${ }^{1}$
}

\section{La lodyans, genre du Rire haïtien}

La littérature orale populaire a toujours été un vecteur de subversion et de critique sociale. En Haïti, où l'anarchie gouvernementale caractérise la situation politique depuis la fondation du pays ${ }^{2}$, il existe un genre spécial pour fustiger et se moquer de lélite administrative : la lodyans (l'audience, du français). Comme l'indique son étymologie, ce bref récit satirique d'origine populaire, fort de sa prise carnavalesque sur la réalité sociopolitique, s'engage à informer la population (l'audience) sur l'actualité au moyen de la parodie et de la dérision. Selon Georges Anglade (2010, p. 18-19), théoricien du genre, la lodyans est la forme esthétique littéraire par excellence du Rire haïtien, c'est-à-dire la manière typiquement haïtienne de répondre aux frustrations et aux complications de la quotidienneté en exorcisant la douleur par l'humour et l'(auto) ironie. Ce genre de l'oralité populaire se fonde sur quelques principes de base qui en font un art de la voix ; parmi ses traits principaux, l’on remarque la brièvetée,

- Sara Del Rossi - doctorante à l'Institut d'Études Romanes de l'Université de Varsovie. Adresse de correspondance : ul. Smyczkowa 9/F603, 02-678 Varsovie, Pologne ; e-mail : saradelrossi88@gmail.com

ORCID iD : https://orcid.org/0000-0003-4897-0236

1. Le présent article a été réalisé grâce à la subvention du projet Dynamika rozwoju haitańskiej opowieści ludowej (n² 2017/27/N/HS2/00675), accordée par Narodowe Centrum Nauki, Polska (Centre National de Recherche Scientifique, Pologne).

2. De nombreuses formes de gouvernement se sont succédé en Haïti, de la république à la dictature, y compris la monarchie et l'empire, entrecoupées par des coups d'État.

3. «[...] tout est suggéré beaucoup plus que développé, connoté plus que noté, allusif. Donner dans la miniature, c'est esquisser en réduisant, c'est pointiller en décrivant, c'est expliquer avec des clins d'œil en mille mots et en moins de dix minutes » (Anglade cité dans Lévy, 2004, p. 207). 
l'ancrage dans le réel $l^{4}$ et l'humour ${ }^{5}$. Par ailleurs, le format " miniature $»^{6}$, propre à la lodyans, permet une fidèle reproduction à l'échelle de la société, laquelle se recompose dans une mosaïque de miniatures dont chacune est consacrée à une thématique donnée. Par conséquent, le récit fictif s'avère être le miroir de l'espace public ; pourtant, sa première fonction n'est pas simplement celle de (re)jouer les saynètes de la vie quotidienne, mais de leur donner une perspective critique. En ce sens, narrer sur un mode ironique ou humoristique les tribulations des classes sociales haitiennes n’a pas une visée uniquement ludique; au contraire, la lodyans a " [...] une fonction normative de voyance d'un monde meilleur » (Anglade, 2004, p. 85), sans pour autant tomber dans le piège des bons sentiments.

\section{La comédie humaine haïtienne de Gary Victor}

À cause de son caractère mordant, la lodyans a été longtemps victime de la censure qui a souvent interdit la publication d'œuvres critiquant le régime politique, en dépit de leur caractère fictif. Toutefois, sa résistance au cours des décennies est due aussi à son indéniable influence sur la littérature haïtienne ${ }^{7}$, tant au niveau du discours narratif que du registre stylistique (Parisot, 2018, p. 252-257). La lodyans joue un rôle fondamental chez le romancier haitien Gary Victor, au point que l'écrivain a été souvent identifié comme le rénovateur et, en même temps, continuateur de ce genre héritier direct de Justin Lhérisson et de Maurice Sixto, les deux maîtres de la lodyans traditionnelle. Comme le souligne Peggy Raffy-Hideux : « Dans la lignée de Maurice Sixto, grand conteur et humoriste haitien, il [Gary Victor] ne cesse de porter un regard acide sur les différents types de pratiques en Haïti, qu'elles soient religieuses, morales, culturelles, politiques, sociales, économiques et même écologiques » (2013, p. 160).

Cette attention portée au présent du pays caractérise toute l'œuvre de Victor, qui est l'un des auteurs les plus lus de nos jours en Haïti. Sa notoriété est due à son succès littéraire, mais aussi à sa collaboration avec la radio, la télévision, le théâtre et le cinéma, sans parler de son engagement dans les affaires politiques et sociales de son

4. "La lodyans est un témoignage puissant des questions qui se posent à chacune des générations " (Anglade cité dans Lévy, 2004, p. 210).

5. «[... la lodyans est un genre du rire, de toute la gamme des rires, de la jovialité à la risette, de la jubilation à la rigolade, et de toutes les déclinaisons des rictus et autres rires jaunes » (Anglade cité dans Lévy, 2004, p. 213).

6. "L'histoire racontée doit avoir en effet toutes les caractéristiques des œuvres de grand format, mais en modèle réduit [...]. La lodyans est ainsi un roman-fleuve en miniature » (Anglade cité dans Lévy, 2004, p. 207).

7. Nous nous référons, en particulier, à Une chercheuse (1888) de Louis-Joseph Janvier, Les chiens (1961) de Francis Joachim Roy, Pays sans chapeau (1997) de Dany Laferrière et God loves Haiti (2015) de Dimitri Elias Léger. 
pays natal (Chemla, 2015, p. 161-185). D'ailleurs, à l'instar de Justin Lhérisson, qui réunissait ses lodyans dans le journal Le Soir, Victor débute sa carrière de lodyanseur dans les pages de Le Nouvelliste. Jusqu'à nos jours, il a consacré plusieurs recueils à la lodyans ${ }^{8}$, sans compter la présence et l'influence de cette dernière dans ses romans $^{9}$ (Cévaër, 2018, p. 58).

Prise dans son ensemble, la production de Victor se veut une vaste fresque de la société haïtienne de la période finale (1983-1989) de la présidence de Jean-Claude Duvalier (dite Jean-Claudisme), considérée comme une caricature du Duvaliérisme (Buteau et Trouillot, 2013, p. 13), jusqu'à l'après-séisme de janvier $2010^{10}$. La profonde connaissance des rouages qui gèrent la machine politique haïtienne est due en partie aux nombreuses expériences personnelles et professionnelles de l'écrivain en tant qu'agronome, fonctionnaire dans l'administration publique et journaliste. Comme le constate Mona Guérin (dans Victor, 1989, p. 8-9) :

Gary Victor possède au bout de ses doigts une plume agile et précise, et au fond de son cœur l'amertume d'un adulte qui en a trop vu, dans sa tête une vision impressionnante de l'universel et du quotidien haitien [...]. [O]n se rend compte à quel point le passage de Gary Victor dans l'Administration Publique l'a profondément choqué et marqué, et on se demande si, sursaturé tout à coup de ce qu'il a vu, il n’a fait que laisser courir sa plume, sa fameuse plume féroce, précoce, et truffée de trouvailles percutantes.

Parmi les figures qui peuplent l'univers fictif de la comédie humaine de Victor, Albert Buron et Sonson Pipirit se détachent de la foule. Ces deux personnages sont devenus emblématiques ; cependant, il est impossible de leur coller une étiquette précise, car l'un trompe l'autre pour son profit et vice-versa (Nzengou-Tayo, 2010, p. 4041). Ils appartiennent à deux milieux sociaux différents, ce qui entraine des controverses entre eux et favorise la figuration des conflits sociaux (Raffy-Hideux, 2013, p. 245-251). Unis par la même volonté de gravir les échelons de la hiérarchie sociale, ils ont pourtant en vue deux objectifs différents : "Tandis que Buron souhaite atteindre la voie de la Présidence pour obtenir le "pouvoir suprême", Pipirit désire

8. Voir à titre d'exemple : Albert Buron ou profil d'une élite. Tome 1 (1989), Sonson Pipiritt ou profil d'un homme du peuple (1989), Histoires entendues et vécues dans un tap-tap (2013) et La queue de Corneille Soisson (2014).

9. Françoise Cévaër se réfère, en particulier, à Clair de Mambo (1990), La piste des sortilèges (1996) et Les cloches de la Brésilienne (2006).

10. Le recueil Histoires entendues et vécues dans un tap-tap (2013) se penche sur les problèmes rattachés au train-train de la vie quotidienne et offre un éventail de situations pénibles auxquelles la population doit faire face chaque jour depuis le séisme de 2010. Le choix du tap-tap (petit autobus urbain) permet à l'auteur de passer en revue les divers types de personnes qui défilent devant les yeux du narrateur-écrivain, chacune ayant à faire à une problématique sociale différente. Parmi les problèmes sociaux abordés, on trouve l'homophobie, la confiance excessive aux charlatans, la croissance de la violence et l'exploitation sexuelle des femmes. 
l'argent et les femmes" (Raffy-Hideux, 2013, p. 270). Chacun protagoniste à son tour d'un recueil de lodyans, ils se font charge des caractéristiques des catégories sociales auxquelles ils appartiennent : l'élite au pouvoir et ses parvenus, les flatteurs versatiles et le peuple. Aussi dans ces portraits Victor opère-t-il une caricature accumulative, une sorte d'énumération des vices qui rongent la société haïtienne.

\section{Albert Buron, I'homme comme il faut}

Le personnage d'Albert Buron naît dans les pages de Le Nouvelliste en 1983, pendant la période dictatoriale. Il est le protagoniste de trois recueils de Victor : Albert Buron ou profil d'une élite, qui se compose de deux tomes édités en 1989 et 1999, et Chroniques d'un leader haïtien comme il faut, les meilleures d'Albert Buron (2006), ce dernier publié au Québec par Mémoire d’encrier. Albert Buron est le représentant de l'élite administrative et politique haïtienne depuis le début du Jean-Claudisme jusqu’à la période chaotique, allant de 1986 à 1989, qui a marqué et suivi sa fin.

Au cours des années, l'image de Buron change au fur et à mesure que la situation sociopolitique haïtienne évolue : il a été un Mulâtre bourgeois, un intellectuel, un employé de l’administration publique et un leader politique. En général, passer aux yeux des autres pour un homme " comme il faut " (Victor, 1989, p. 37) est sa seule stratégie pour assouvir son désir de gravir peu à peu tous les échelons de la hiérarchie sociale jusqu’à la Présidence, en s'adaptant chaque fois aux changements sociopolitiques. Ainsi, dans le but de réussir son escalade sociale, il fait preuve d'un transformisme politique qui l'amène à glisser du communisme progressiste ${ }^{11}$ - propre à l'intellectuel de la Négritude - au fascisme duvaliériste ${ }^{12}$, tout en embrassant les idéologies des divers partis politiques nés après la fin de la dictature des Duvalier, en particulier le Parti National Progressiste (PNP), analogue fictionnel du Rassemblement des démocrates nationaux progressistes (RDNP) du démocrate-chrétien Leslie Manigat ${ }^{13}$.

Toutefois, le caméléonisme idéologique d'Albert Buron est compensé par certains traits invariables qui permettent à Victor de parodier les caractéristiques de la classe moyenne haïtienne des années 1980, composée principalement de parvenus arrivistes et corrompus. Ainsi, l'auteur charge (carica) Albert Buron des vices et des travers de sa couche sociale et fait dérouler ses aventures dans un univers d'" [...] arrogance, désinvolture, suffisance, mépris des autres, impudence, hypocrisie foncière, esprit

11. "J’ai défendu avec panache les valeurs de l’Homme Noir. Je me suis sacrifié pour la Négritude, pour l'Égalité et pour la Fraternité. Tout par et pour le peuple ! Voici ma devise, Phil ! (Victor, 1989, p. 10).

12. «Il n'y a que le communisme qu'il faut interdire à tout prix dans une société pluraliste. [...] Le duvaliérisme n'est pas du fascisme » (Victor, 1989, p. 118).

13. Figure énigmatique de la vie politique haïtienne, Leslie Manigat a été d'abord supporteur et collaborateur de François Duvalier, qui l'a ensuite condamné à l'exil dans les années 1960 ; en 1988, il est élu Président d'Haïti grâce au soutien des forces armées. 
de corruption, de clan, de classe et de chapelle (Guérin cité dans Victor, 1989, p. 7).

La première attitude est nommée par Victor «m’as-tu-vu» (1989, p. 20). Il s'agit de la manie, propre à l'individu Jean-Claudiste, de manifester le prestige de sa propre condition sociale à travers un étalage de luxe effréné (Buteau et Trouillot, 2013, p. 17-18) ; ainsi, la première communion du fils de Buron devient le prétexte pour une orgie de surabondance matérielle, mise en relief par l'emploi de l'accumulation dans la description :

Quand Buron se met en tête de réaliser quelque chose de grandiose, mon cher ami, il n'hésite pas à puiser dans les fonds de l'État. Laisse-moi te donner un petit aperçu des moyens mis en ouvre pour épater la galerie. Cortège de trois Mercedes [...], serveurs en livrée de luxe, tout le staff de Carré-Virgule, l'un de nos restaurants les mieux cotés, [...] un véhicule affrété uniquement pour le transport continu des boissons et de la nourriture, et j'en passe... Tiens... J'oubliais : [...] Tout était en verre, en porcelaine et en argent. (Victor, 1989, p. 22)

L’extrait introduit la deuxième caractérisation incarnée par Buron : l'aloufas (glouton, en créole haïtien). Le terme traduit le pillage effréné pratiqué par de nombreux membres de lélite politique et administrative qui engouffrent les fonds publics au moyen de la corruption et de pratiques malhonnêtes (contrebande, prostitution, chantage, trafics de déchets, de drogue et d'organes). La caricature, choisie comme image de couverture du recueil, résume la conduite louche et ambivalente des technocrates et des politiciens, censés agir pour le bien du pays :

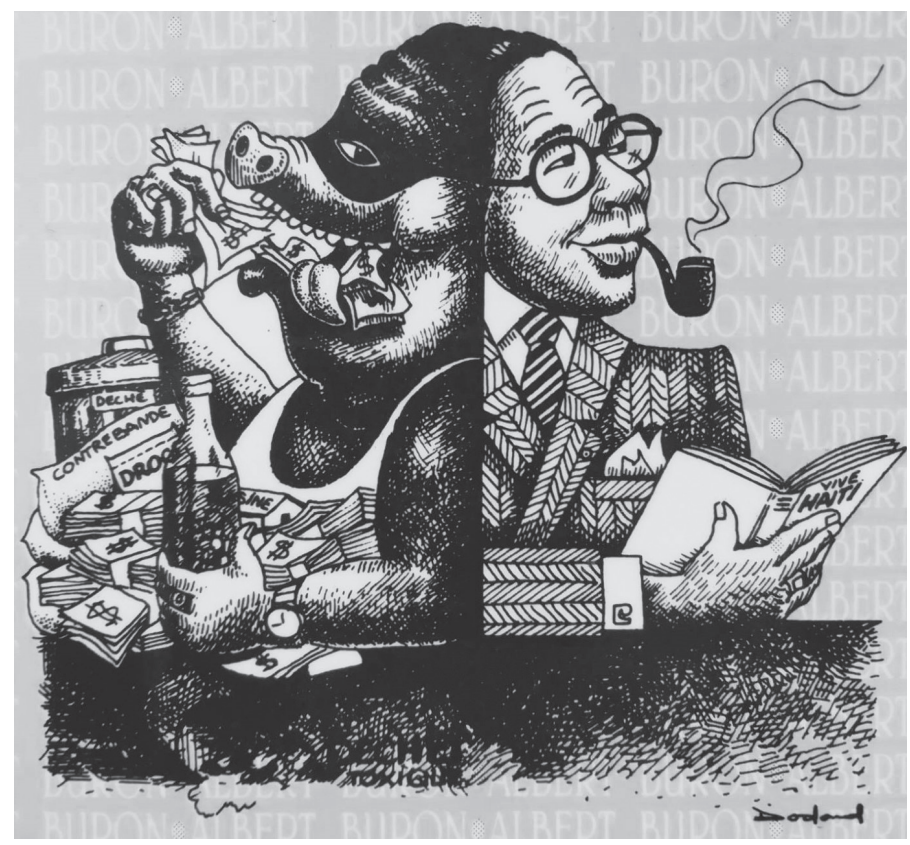

Figure 1. Caricature d'Albert Buron par Philippe Dodard (Victor, 1989, page de couverture) 
L'insatiabilité et l'égoïsme dictent la discipline de ces hommes comme il faut qui, depuis toujours, obéissent aux préceptes de la corruption institutionnalisée. Comme en témoigne Buron lui-même, " [l]e monde est comme il faut. Il y a ceux qui mangent et ceux qui se font manger. [...] Les gens comme il faut sont ceux qui laissent les choses telles qu'elles sont. Les gens honnêtes sont la calamité de ce siècle » (Victor, 1989, p. 37). Il se proclame aussi théoricien du sétoupamisme, principe qui résume la conduite des hommes comme il faut :

Le sétoupamisme est un terme créé à partir de l'expression créole se tou pa m', qui veut dire : à mon tour maintenant. [...] Le sétoupamisme vise au bien-être de l'homme et il n'y a qu'une seule chose à faire pour obtenir ce bien-être : remplacer ceux qui jouissent déjà de ce bien-être. (Victor, 1989, p. 213-215)

Au niveau politique, il s'agit d'obtenir un rendement maximal à moindre frais et de s'enfuir à l'étranger avec sa fortune (à l'instar de Jean-Claude Duvalier ${ }^{14}$ ). Le sétoupamisme ne se limite pas seulement à la classe politique, il est aussi révélateur de la "psychologie haitienne » (Victor, 1989, p. 215) qui se satisfait des besoins du moment sans aucune prévention et qui gangrène toutes les couches sociales. Selon Victor, ce système n'est réalisable qu'avec l'appui de la population qui ne réagit pas aux abus du gouvernement et cherche, elle aussi, à profiter de la situation :

La marchande de fritures sétoupamiste dépensera des sommes appréciables pour trouver un charme qui lui permette de supplanter la marchande du quartier préférée de la clientèle. Dans l'Administration Publique, les cadres supérieurs sont en majorité sétoupamistes, car ils œuvrent continuellement à déchouquer [déboulonner] les chefs de service, les assistants-directeurs, les directeurs, les ministres, etc. (Victor, 1989, p. 214)

\section{Sonson Pipirit, I'homme du peuple}

Sonson Pipirit est un homme du peuple, fier représentant de la psychologie haïtienne, il symbolise tous ceux qui tirent profit de la situation et de l'orientation politique les plus favorables. Dans le recueil Sonson Pipirit ou profil d'un homme du peuple (1989), on assiste aux nombreuses étapes de sa carrière : orphelin, restawè ${ }^{15}$, gigolo, macoute ${ }^{16}$,

14. Lors de sa fuite-exil en France, Jean-Claude Duvalier disposait d'une grosse fortune (entre 300 et 800 millions de dollars) soustraite aux caisses de l'État.

15. Enfant placé chez une famille autre que la sienne (il s'agit souvent d'une forme contemporaine d'esclavage).

16. Milicien, membre de la milice paramilitaire d'un gouvernement autoritaire. 
déchouketeur ${ }^{17}$, communiste, policier, bòk $\dot{o}^{18}$, jusqu'à devenir millionnaire, avant de se retrouver à nouveau pauvre. Les (més)aventures de ce picaro, comme le définit Raffy-Hideux (2013, p. 270), et son escalade sociale permettent à Victor de dresser le portrait de toutes les couches de la population, à partir de l'échelon le plus bas.

Ainsi, avec Pipirit, l'on quitte les cabinets des technocrates et les salons de la bourgeoisie pour arpenter les ruelles des bidonvilles de Port-au-Prince. Ici, l'insalubrité domine sur un océan de «[...] boue épaisse, gluante, presque verdâtre qui pouvait peut-être servir de matière première pour la fabrication d'une bombe bactériologique " (Victor, 1989a, p. 52). La pauvreté, le manque d'hygiène, la famine et le désespoir triomphent dans le ventre de Port-au-Prince, décrit comme un immense «no man's land» (Victor, 1989a, p. 53) habité par ceux qui ont perdu toute trace d'humanité.

Gary Victor fait de Pipirit le lien entre le bas et le haut, puisqu'il assume une fonction double et ambivalente, celle d'un serviteur qui se sert à son tour du maitre. Que ce soit comme restawèk-gigolo de la femme d'un ministre ou leader d'une foule payée pour soutenir la promotion d'Albert Buron à la direction d'un ministère, Pipirit tire toujours profit de son infériorité supposée. Aussi la ridiculisation des couches sociales se réalise-t-elle par le dévoilement de secrets et d'aspects déplaisants. Grâce à Pipirit, l'on assiste à une parade de personnages caricaturés, des figures inoubliables qui montrent les vices de la société haïtienne : une nymphomane, fondatrice d'une secte, qui communique avec le Christ à travers une couleuvre ; un professeur de collège, ancien cancre, qui a obtenu son poste grâce à son expérience vicennale de macoute ; un juge de paix, «[...] auteur d'une série de procès-verbaux qui pourraient ridiculiser à tout jamais la justice haïtienne " (Victor, 1989a, p. 77); un intellectuel qui, à cause d'un virus intestinal, renie son savoir et cède aux superstitions et aux remèdes populaires; et, enfin, un cortège de policiers, politiciens, technocrates, prêtres et commerçants tous corrompus.

Toutefois, la relation entre Pipirit et ses partenaires d'affaire n'est pas mutuelle ; au contraire, il semble toujours avoir une position de dominance. Contrairement à la figure classique du valet rusé, qui dupe le maître au moyen de son astuce, Pipirit a surtout recours à la violence et à la vulnérabilité des autres aux superstitions, les mêmes armes de dissuasion que celles utilisées par François Duvalier pour assujettir la population haïtienne pendant sa dictature. En effet, même si la plupart des histoires de Pipirit se déroulent à la fin et après le Jean-Claudisme, les nombreuses références à Papa Doc et à ses méthodes d'oppression démontrent que le fantasme du Président-à-vie hante encore la société haïtienne. D’ailleurs, celle-ci est encore régie par les rouages de l'époque dictatoriale, même si l'hypocrisie oblige désormais à en changer les mots-clés. À titre d'exemple, le sombre autoportrait donné

17. Organisateur ou participant à un déchoucage, c'est-à-dire une révolte populaire qui se caractérise par le pillage des biens privés des responsables politiques, administratifs et militaires.

18. Prêtre vaudou responsable des zombifications. 
par Boukan Dife nous montre comment le macoutisme se cache derrière la nouvelle terminologie :

Je suis Boukan Dife, roi des marchés, terreur des Recherches Criminelles en tant qu'expert dans le maniement du kokomakak [i.e. maillet] à clous. Je suis un attaché mais je ne suis pas un makout. [...] Je suis tout simplement un chef. Démocratie ou pas, les Haitiens auront toujours des chefs. [...] Le magistrat m’a donné plein pouvoir pour mettre de l'ordre dans cette capitale investie par la vermine qui fuit la province. [...] J'arrête, je tue et je protège. Le truc est bien simple. (Victor, 1989a, p. 143)

Des micro-(auto)portraits satiriques similaires s'enchaînent tout au long du recueil. L'humour noir de Victor réussit à rendre le climat de violence hors contrôle de l'après-dictature. Pourtant, selon l'auteur, il n'y a que deux réactions possibles à une telle situation : l'asservissement muet ou le déchoucage (déboulonnement, en créole haïtien).

Le déchoucage est une révolte populaire qui vise au renversement de la situation politique à travers le pillage et la destruction des biens matériels appartenant aux personnalités au pouvoir et à leurs acolytes. Victor fait de Sonson Pipirit un déchouqueur modèle, au point qu'il devient le propriétaire d'une entreprise spécialisée en services de déchoucage : «[...] déchoucage de ministres, de directeurs généraux, de directeurs, et même de journalistes dans les médias d'État » (Victor, 1989a, p. 56). Généralement associées au peuple, Victor dénonce le fait que ces réactions jugées anarchiques sont souvent commandées par les chefs des partis d'opposition. Le déchoucage s'avère donc être la mise en pratique du sétoupamisme théorisé par Albert Buron; de cette manière, Victor dévoile une fois de plus le système de connexions et de corruption qui maintient en équilibre (précaire) la société haïtienne.

Devenu le bras armé au service des tenants du pouvoir, Pipirit agit toujours dans son propre intérêt, sans se soucier de l'avenir des autres et tournant le dos à ses origines. À cause de son indifférence et de son égoïsme, la population des bidonvilles de Port-au-Prince l'accuse d'être le responsable de l'extrême dénuement de ces quartiers et décharge sa rage sur lui :

Une dizaine de choses s'abattirent sur lui avec voracité. Il perdit connaissance après avoir vaguement compris qu’on le dépouillait de ses cinq cents gourdes. [...] Sonson Pipirit comprit alors que la seule chose à faire était de retourner chez lui [...]. Mais à l'endroit où sélevait sa pyès kay [pièce de maison], il ne restait que quelques tôles tordues et des morceaux de carton sur lesquels des humains sétaient soulagés les intestins. Depuis ce matin-là, Sonson Pipirit quitta la cité et n'y revint jamais. (Victor, 1989a, p. 100-101)

Victime de son propre destin et déchouqué à son tour, Pipirit est contraint à l'exil, d'où il reviendra après une longue période. C'est à ce moment-là que l'existence de ce personnage prend une tournure imprévisible : rongé par des remords de conscience, 
il veut faire le bien. Passé au statut de millionnaire grâce au génie Azimbidim Orikal, il réalise tous ses rêves de richesse et ses désirs les plus voluptueux, entouré par l'élite sociale haïtienne ; pourtant, il est déçu par la bonne société port-au-princienne qui préfère fréquenter le Cercle médiocre d'un Mulâtre, au lieu de son Cercle du dernier cri, vu qu'il reste un Noir qui parle mal le français. Conscient que le préjugé de couleur ne peut pas être effacé par l'argent, Pipirit décide d’agir enfin pour le bien de ses compatriotes. Le dialogue entre Pipirit et son génie maléfique témoigne de la radicalisation de l'égoïsme haïtien :

- Je sais que je possède de l'or à n'en savoir que faire, Azimbidim Orikal. Pourtant quand je vois ces bidonvilles, ces montagnes de détritus, tous ces gens pauvres qui m'assaillent dès que je vais au centre-ville, je me sens dégoûté. [...]

- Change de chemin, Maître... Évite le centre-ville. Fais teinter les vitres de tes véhicules. Ce n'est pas toi qui changeras les choses dans ce pays. [...]

- Mais je suis riche, Azimbidim Orikal... Je peux faire construire des autoroutes, des cités, des écoles, des ponts. [....]

- Maître ! On ne t’a pas donné cette richesse pour faire le Bien. Si Azimbidim Orikal était un esprit du Bien, il ne serait pas ici, en Haïti. [...] [S] ache que le Mal est la seule force qui contrôle cette partie de l'île. [...]

- Que dois-je faire alors, Azimbidim?

- Fais comme les autres, Maitre. Si tu es fatigué avec les femmes, essaye les hommes. Il y a la cocaïne et d'autres plaisirs que tu peux t'offrir. Paroles d'Azimbidim Orikal. (Victor, 1989a, p. 130-131)

La rédemption de Pipirit s'avère être alors un escamotage de Victor pour démontrer, une fois de plus, l'atavisme de la psychologie haïtienne. Le néo-millionnaire, pris par une profonde sensation "dêtre inutile dans une société moribonde " (Victor, 1989a, p. 136), est puni par les esprits maléfiques pour ses désirs philanthropiques. Victor dresse le portrait final de son picaro dépouillé de tous ses biens :

Quand je m’approchai, je fus stupéfait de découvrir un homme, vêtu de guenilles, les pieds nus, qui effectuait cette curieuse danse qui consistait à se contorsionner le corps, parfois au ralenti, parfois avec des mouvements si saccadés qu'on croyait que la tête ou un membre allait s’arracher. [...] Quand il eut terminé son numéro, je m’approchai de lui. Il ne me reconnut pas en dépit de toutes mes tentatives. Ses regards, vides d'expression, semblaient perdus dans un monde qu'il était le seul à connaître. (Victor, 1989a, p. 137-138)

Poussé jusqu’à la folie par l'absurdité du système social haïtien, Pipirit termine son aventure victime du même système qu'il a servi et dont il s'est servi tout au long de sa vie. 


\section{Conclusion}

La société haïtienne et ses figures symboliques sont au centre de tous les ouvrages de Gary Victor, dont la prolificité, l'ironie du regard aigu et le projet littéraire renvoient aisément à l'œuvre d'Honoré de Balzac. À l'instar de Balzac et d'autres écrivains qui ont interrogé et observé le délicat système sociopolitique de leurs sociétés, les portraits et les situations dressés tout au long de la comédie humaine de Victor possèdent une valeur d'anticipation et d'avertissement. En effet, la caricature accumulative utilisée pour le personnage de Buron permet de résumer et dénoncer les abus de l'élite au pouvoir dans les années 1980, dont les actions et les descriptions restent toujours actuelles. De même, la triste parabole par étapes de Pipirit, dévoré par les remords de son passé violent et réduit à la folie, témoigne de l'impossibilité d'une vision optimiste ou d'une quelconque amélioration. Enfin, les nombreuses figures qui peuplent l'univers (semi)fictif de Victor ne font que pousser à l'extrême la caricature de la société haïtienne, dont les aspects ridicules et déplaisants sont mis à nu. Le style mordant de ces portraits-charge et l'humour qui en découle sont donc indispensables pour balancer la cruauté de la réalité décrite. Toutefois, la lodyans de Victor n'est pas seulement un récit raconté sous forme d'anecdote pour égayer ou intriguer l'audience, il ne repose pas non plus uniquement sur l'humour ou sur l'exagération ; il s'agit plutôt d'une façon spécifiquement haïtienne de regarder le monde pour dénoncer, de manière plus ou moins voilée, la réalité du pays. Une telle interprétation réaliste confirme l'engagement de Victor, dont le but est de provoquer un rire jaune conscient des problématiques sociopolitiques du pays.

\section{RÉFÉRENCES}

Anglade, G. (2004). Les Lodyanseurs du Soir : Il y a 100 ans, le passage à l'écrit. Dans M.-A. Sourieau et K. Balutansky (dir.), Écrire en pays assiégé / Haïti - Writing Under Siege (p. 61-87). Leiden / Boston : Brill / Rodopi.

Anglade, G. (2010). Le secret du dynamisme littéraire haïtien. Port-au-Prince : Éditions de l'Université d'État d'Haïti.

Buteau, P. et Trouillot, L. (dir.). (2013). Le prix du Jean-Claudisme. Arbitraire, parodie, désocialisation. Port-au-Prince : C3 Éditions.

Cévaër, F. (2018). Gary Victor : Profile of a Writer for the People. Intertexts, 22(1-2), 55-92. https://muse.jhu.edu/article/743209

Chemla, Y. (2015). Littérature haïtienne 1980-2015. Port-au-Prince : C3 Éditions.

Lévy, J. (2004). Entretiens avec Georges Anglade. L'espace d’une génération. Montréal : Liber.

Nzengou-Tayo, M.-J. (2010). The Haitian Short Story : An Overview. Journal of Caribbean Literatures, 6(3), 37-52. https://www.jstor.org/stable/41411860

Parisot, Y. (2018). Regards littéraires haitiens. Cristallisations de la fiction-monde. Paris : Classiques Garnier. 
Raffy-Hideux, P. (2013). Les Réalismes haïtiens contemporains. Récit et conscience sociale. Paris : Honoré Champion.

Victor, G. (1989). Albert Buron ou profil d'une élite. Tome 1. Port-au-Prince : Éditions Henri Deschamps.

Victor, G. (1989a). Sonson Pipiritt ou profil d'un homme du peuple. Port-au-Prince : Éditions Henri Deschamps.

\section{FIGURE :}

Dodard, Ph. (1989). Caricature d'Albert Buron. Dans G. Victor, Albert Buron ou profil d'une élite. Tome 1. (page de couverture). Port-au-Prince : Éditions Henri Deschamps.

RÉSUMÉ : La littérature orale populaire a toujours été le moyen le plus efficace pour critiquer le pouvoir. En Haïti, il existe un genre spécial pour fustiger et se moquer de l'élite administrative : la lodyans. Ce bref récit satirique d'origine populaire, fort de sa prise carnavalesque sur la réalité sociopolitique, est utilisé pour informer la population sur l'actualité à travers la parodie et la dérision. Cet article se propose d'analyser l'utilisation de la caricature accumulative dans les portraits sociaux des lodyans de Gary Victor. Les deux personnages principaux, Albert Buron et Sonson Pipirit, peuvent être considérés comme des types sociaux emblématiques, qui se font charge des caractéristiques des catégories sociales auxquelles ils appartiennent. La narration des (més)aventures de ces deux personnages et des figures qu'ils rencontrent tout au long de leurs parcours permet à Victor de dresser une sorte de comédie humaine haïtienne, très proche de la réalité. Une telle interprétation réaliste confirme le caractère engagé de l'auteur, dont le but est de provoquer un rire jaune conscient des problématiques sociopolitiques du pays.

Mots-clés : lodyans, littérature haïtienne, Gary Victor, caricature

\section{The caricatural lodyans in Gary Victor's Haitian Human Comedy}

ABSTRACT: Popular oral literature has always been the most effective means of criticizing authority. In Haiti, there is a special genre to castigate and mock the administrative elite: the lodyans. This short satirical tale of popular origin, with its carnivalesque grip on socio-political reality, is used to inform the population about current events through parody and derision. This essay aims to analyze the use of the accumulative caricature in the social portraits in Gary Victor's lodyans. The two main characters, Albert Buron and Sonson Pipirit, can be considered emblematic social types, who embody the main traits of their social categories. The narration of their (mis)adventures and the portraits of the characters met through their stories allow to draw 
up a sort of Haitian Human Comedy, so nigh to reality. This type of interpretation confirms Victor's engagement, whose aim is to provoke a forced laughter, aware of Haitian sociopolitical issues.

Keywords: lodyans, Haitian literature, Gary Victor, caricature 